\title{
Predictive Value of Contrast-Enhanced Ultrasound in Chemical Lumbar Sympathectomy for End-Stage Arteriosclerosis Obliterans of the Lower Extremities
}

\author{
Yue Wu - Suming Tian · Chunye Li $\cdot$ Weibo Zhang · Qianqian Xing · \\ Gang Chen
}

Received: November 2, 2021 / Accepted: December 10, 2021 / Published online: January 15, 2022

(C) The Author(s) 2022

\begin{abstract}
Introduction: The therapeutic effect of chemical lumbar sympathectomy (CLS) on ischemic diseases of the lower limbs varies greatly among individuals. The time to peak (TTP) response in contrast-enhanced ultrasound (CEUS) can reflect the perfusion disorder of the calf skeletal muscle and the collateral circulation. In this study we evaluated the predictive value of CEUS in patients treated with CLS for end-stage atherosclerotic occlusive disease of the lower extremity (ASO-LE).

Methods: This was a prospective study that included patients with end-stage ASO-LE and moderate to severe pain who had undergone a CEUS examination and CLS procedure and who were observed for 12 months after surgery. The patients' characteristics and prognostic factors,
\end{abstract}

Supplementary Information The online version contains supplementary material available at https:// doi.org/10.1007/s40122-021-00347-9.

Y. Wu · S. Tian · W. Zhang · Q. Xing · G. Chen ( $₫)$ Department of Anesthesiology, Sir Run Run Shaw Hospital affiliated with School of Medicine of Zhejang University, 3 Qingchun Road East, Shangcheng District, Hangzhou 310016, Zhejiang, People's Republic of China e-mail: chengang120@zju.edu.cn

C. Li

Department of Pain, Affiliated Hospital of Jiangsu University, Zhenjiang 212001, Jiangsu, People's Republic of China including lower limb pain score, skin temperature, walking distance, and ulcer and gangrene healing, were recorded.

Results: Fifty-eight patients with a mean age of 66.24 (range 58-78) years were included in the study, of whom $42(71.41 \%)$ were men. Following the CLS procedure, the numerical rating scale (NRS)-measured pain decreased significantly, and the skin temperature of the affected limb increased significantly $(P<0.05)$. The satisfaction rate of lower limb pain relief 1 year after operation was $53.45 \%$. Correlation analysis showed that preoperative TTP response was correlated with the NRS score and skin temperature of the affected limb at 6 months and 12 months post surgery $(P<0.05)$. The binary logistic regression analysis indicated that a longer preoperative TTP response was associated with a higher risk of poor pain relief after CLS (odds ratio 1.126, 95\% confidence interval 1.058-1.205). The receiver operating characteristic curve showed that preoperative TTP response had a certain predictive value on CLS treatment effect, with a sensitivity and specificity of $81.5 \%$ and $83.9 \%$, respectively. When the preoperative TTP response was $>77.5$, the risk of poor response after CLS increased.

Conclusions: Preoperative TTP response was able to predict the therapeutic effect of CLS to a certain extent, and thus may aid physicians in determining the choice of CLS treatment for patients with ASO-LE. 
Trial Registration: Chinese Clinical Trial Registry: ChicTR1900028424 (principal investigator: Yue $\mathrm{Wu}$; date of registration: 21 December 2019).

Keywords: The time to peak; Peripheral arterial disease; Arteriosclerosis obliterans; Skeletal muscle; Collateral circulation

\section{Key Summary Points}

Why carry out this study?

Chemical lumbar sympathectomy (CLS) is the main alternative treatmentfor severe ischemic lower extremity diseases, but the therapeutic effect remains uncertain. Contrast-enhanced ultrasound (CEUS) of the calf skeletal muscle allows assessment of the perfusion level of the lower extremities. We assume that the time to peak (TTP) obtained from CEUS can predict the effect of CLS on end-stage atherosclerotic occlusive disease of the lower extremity.

\section{What was learned from the study?}

The results suggest that preoperative TTP response was correlated with pain score and skin temperature of the affected limb after CLS, indicating that a longer preoperative TTP was associated with a higher risk of poor pain relief after the operation and leading to the conclusion that that preoperative TTP can predict the therapeutic effect of CLS to a certain extent.

In general, the predictive effect of CEUS seems to be reliable, but the results should be interpreted with caution.

\section{INTRODUCTION}

Arteriosclerosis obliterans (ASO) is the main cause of peripheral arterial disease (PAD). With lifestyle changes and an aging population, the prevalence of PAD has increased significantly in recent years. The overall prevalence of PAD is $3-10 \%$ in the general population, but it increases to $15-20 \%$ among people older than 70 years [1]. As the disease progresses, patients with ASO of the lower extremity (ASO-LE) have intermittent claudication and even critical limb ischemia (CLI), which manifests as resting pain, refractory ulcers, and gangrene in the lower extremities.

For patients with ASO-LE and CLI, the main treatment is revascularization through percutaneous transluminal angioplasty or surgical bypass. However, because of unfavorable vascular involvement or high surgical risk, as many as one-third of patients are not suitable for revascularization [2, 3]. Even after vascular reconstruction surgery, the long-term prognosis is poor, and the postoperative restenosis rate is high-potentially reaching $40-60 \%$ after 1 year [4].

For patients with end-stage ASO-LE and poor treatment outcomes, chemical lumbar sympathectomy (CLS) can achieve a substantial therapeutic effect $[5,6]$. The lumbar sympathetic nerve is involved in regulating the vasomotor function of the lower limbs. CLS induces damage to the lumbar sympathetic nerve through nerve disruptors, expands the spasm blood vessels of the lower limbs, and increases collateral circulation, ultimately achieving the purpose of improving the peripheral blood supply and promoting the healing of local ulcers $[5,6]$. CLS is completed under computed tomography (CT) guidance. It has the advantages of being a safe and minimally invasive procedure, and it is a simple operation, with repeatable implementation, and is relatively economical and well accepted by patients.

In our previous study we reported that the efficacy of CLS treatment was $50-60 \%$ at 6 months. Patients with poor treatment success were mainly those with refractory ulcers and severe gangrene of the lower extremities. These patients have extremely poor lower extremity peripheral circulatory functions, as noted in previous research reports $[7,8]$. The treatment effect of CLS should be closely related to the severity of lower limb peripheral perfusion. 
Therefore, an objective indicator that can effectively predict the effect of CLS treatment is needed to avoid unnecessary trauma and the misappropriation of medical resources caused by using CLS in an inappropriate patient population.

Contrast-enhanced ultrasound (CEUS) is a new technology for evaluating perfusion imaging. Through intravenous injection of an enhanced contrast agent, CEUS provides a variety of quantitative parameters beyond those available from conventional ultrasound examinations, and the time to peak (TTP) parameter can be used an a noninvasive and objective measure to evaluate the level of skeletal muscle perfusion and collateral circulation caused by ischemic diseases in the lower extremities [9-13].

At the present time, the perfusion level of ischemic skeletal muscle cannot be directly evaluated by color Doppler ultrasound, CT, magnetic resonance imaging (MRI), and digital subtraction angiography. Although positron emission tomography and contrast-enhanced MRI can be used to assess the level of skeletal muscle perfusion, they are expensive and timeconsuming procedures and are not suitable for routine examinations [14, 15]. CEUS has the advantages of being real-time, convenient, repeatable, and non-radiation-based assessment tool, and it meets many requirements for routine patient screening, such as low cost, convenient transport, and short appointment time.

Whether the therapeutic effect of CLS can be predicted by CEUS has not yet been determined. In this study, we hypothesized that CEUS can be used to predict the therapeutic effect of CLS on patients with end-stage ASO-LE. We assessed response by measuring changes in lower limb pain and skin temperature, the number and distance of patients able to walk, and the healing time of skin ulcers.

\section{METHODS}

\section{Study Population and Design}

This study was performed in accordance with the Helsinki Declaration of 1964, as revised in
2013, and its protocol was approved by the Institutional Review Committee of Run Run Shaw Hospital of Zhejiang University in Hangzhou, Zhejiang Province, China. Data collection was pre-planned and registered with the Chinese Clinical Trial Registry (Registration number: ChicTR1900028424). All participants were informed about the risks and complications before surgery, and all patients provided written informed consent.

Patients with end-stage ASO-LE (Fontaine III and IV) who had moderate and severe pain ( $\geq 4$ on a numerical rating scale [NRS] [16]) were included in this study. All patients may or may not have been treated with intracavitary interventional surgery (balloon angioplasty or carotid stenting). Exclusion criteria were: (1) a history of chronic pain and mental illness; (2) vascular interventional surgery during postoperative follow-up; (3) severe hepatic and renal insufficiency and poor cardiopulmonary function; (4) systemic or low back infections; (5) abnormal platelet function or coagulation dysfunction caused by long-term oral antiplatelet or anticoagulant drugs; (6) inability to tolerate the prone position; (7) allergy to anhydrous ethanol and iodohexanol; (8) implantation of cardiac pacemakers; and (9) unwilling to participate in the experiment.

\section{CEUS Examination and Image Analysis}

\section{Instrument and Setup}

For CEUS, Philips' Epiq 5 shared service ultrasound system was used (Philips Healthcare, Bothell, WA, USA), which included a line array probe with scan frequencies of $6-10 \mathrm{MHz}$. The settings used were: mechanical index, 0.08; sound output, $5 \%$; depth, $4 \mathrm{~cm}$; single focus at $3.75 \mathrm{~cm}$; frequency, $6 \mathrm{MHz}$; gain, 36; and measurement unit, in millimeters.

\section{Test Methods}

The CEUS procedure was conducted $10 \mathrm{~min}$ after the patient had rested in a supine position, and the probe was placed in the central part of the skeletal muscle of the calf, in the medial part of the knee joint. The skeletal muscle of the posterior calf region was selected as the research 
location because it is one of the main plantar flexor muscles of the lower limb, is superficially located, is at a consistent anatomical position, and has an extensive anastomotic vascular network [17]. SonoVue contrast agent (Bracco Suisse SA, Plan-Les-Ouates, Switzerland) was used, and $5 \mathrm{~mL}$ of saline (Otsuka Pharmaceutical Co., Ltd. Tianjing, China) was added; the mixture was vibrated to cause dissolution into a microbubble suspension. An intravenous indwelling needle with a three-way tube was placed into the left anterior elbow vein, and $2.4 \mathrm{~mL}$ of the suspension was was injected rapidly, following which the tube was flushed with $5 \mathrm{~mL}$ of normal saline at the same speed, and angiographic images were obtained for $4 \mathrm{~min}$. The probe was fixed to keep the two-dimensional image unchanged. The image was saved on a hard disk. All patients underwent CEUS examination 1 day before and 1 month after CLS.

\section{Image Analysis}

QLAB quantification software (Philips Investment Co., Ltd., Bothell, WA, USA) was used to analyze the changes in the echo volume of each pixel and in the contrast agent in the skeletal muscle (gastrecnemius and part of soleus) of the lower leg. The results were used to obtain the contrast time intensity curve (TIC); the selection of the region of interest should attempt to completely cover the skeletal muscle (gastrecnemius and part of soleus) in the ultrasound section. The characteristics of blood flow dynamics in the target area were analyzed by the TIC (Fig. 1), and CEUS perfusion parameters were obtained. The curve shape of the enhancement phase and the TTP were analyzed. CEUS was performed by an experienced physician, and the images were analyzed by two experienced physicians using a double-blind method. All quantitative indicators, including TTP, were calculated using the 'local density random walk wash-in wash-out' function. The TTP was averaged from three measurements to reduce errors.

\section{Chemical Lumbar Sympathectomy}

Chemical lumbar sympathectomy was performed with the patient in the prone position and was guided by CT scan. The temperature in the CT operating room was controlled at $25 \pm 1{ }^{\circ} \mathrm{C}$. We selected the middle and lower third of the L2 vertebra to the middle and upper third of the L3 vertebra, as well as the anterolateral margin of both vertebrae as the puncture point [18]. To achieve greater longitudinal diffusion of the drug and more complete destruction, we used a two-needle method for puncture (Fig. 2). First, $3 \mathrm{~mL}$ of $2 \%$ lidocaine (Shandong Hualu Pharmaceutical Co., Ltd. Chiping County, Shandong Province, China) containing iodohexanol (Yangtze River Pharmaceutical Co., Ltd. Taizhou City, Jiangsu Province, China) was injected into each puncture point. A CT scan confirmed that the contrast agent was distributed in the anterolateral part of the second or third lumbar vertebra and in the anterior edge of the psoas major muscle, indicating that the puncture needle and drug were accurately distributed. If the patient had no numbness in the groin area or lower limbs or had no decreased muscle strength in the lower limbs, $2.5 \mathrm{~mL}$ of anhydrous ethanol (Anhui Ante Co., Ltd., Anhui, China) was slowly injected $20 \mathrm{~min}$ later. CLS was always performed by the same team of experienced physicians.

\section{Observations and Follow-Up}

Patient demographics and clinical data were recorded and collected; data included gender, age, body mass index (BMI, in $\mathrm{kg} / \mathrm{m}^{2}$ ), history of smoking, history of ulcers or gangrene, history of vascular interventional surgery, comorbid disease, ankle-brachial index (ABI), surgical side, preoperative pain duration, TTP, pain score, and dosage of tramadol (Beijing Mendi Pharmaceutical Co., Ltd, Beijing, China). TTP was recorded preoperatively and 1 month postoperatively; measures were obtained by CEUS examination of the skeletal muscle of the affected leg.

The foot pain score, skin temperature, 6-min walk test result, and ulcer healing were recorded 


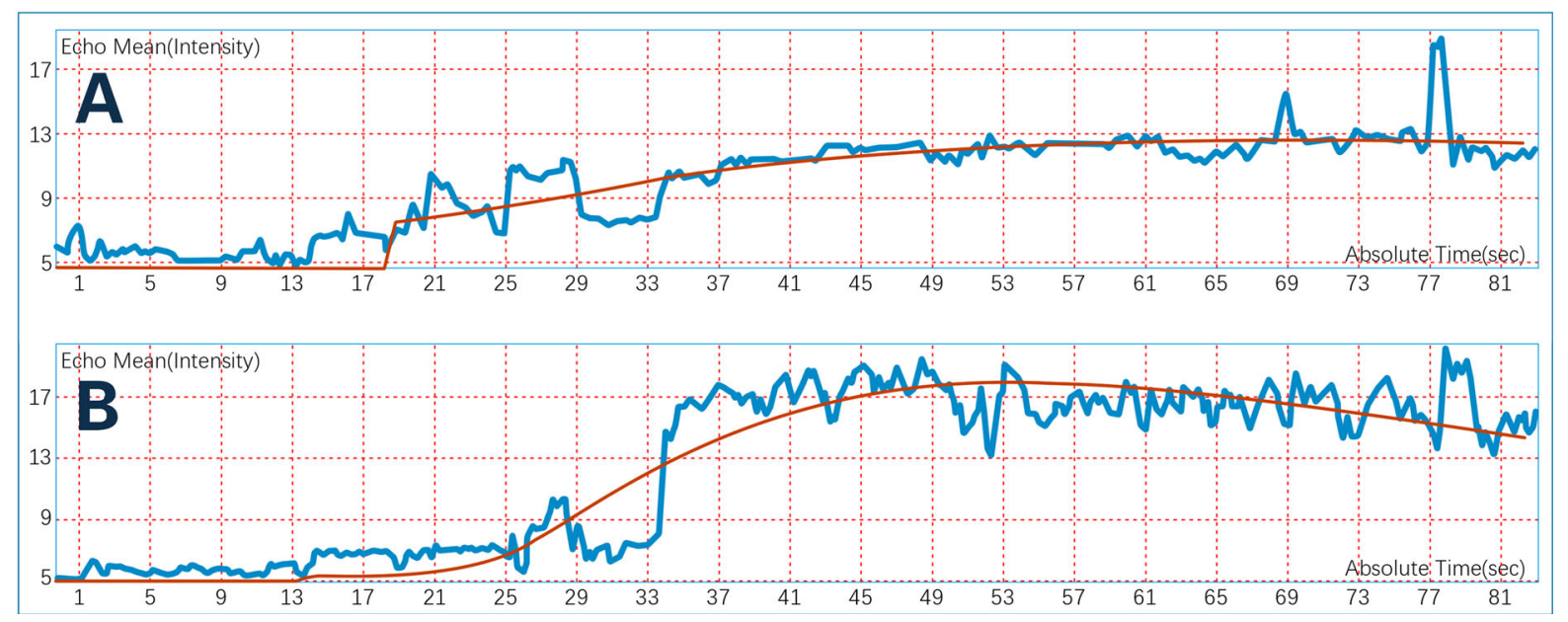

Fig. 1 Time-intensity curve of contrast-enhanced ultrasound imaging. The calf skeletal muscle with a diameter of $1.5 \mathrm{~cm}$ was taken as the area of interest. a, b

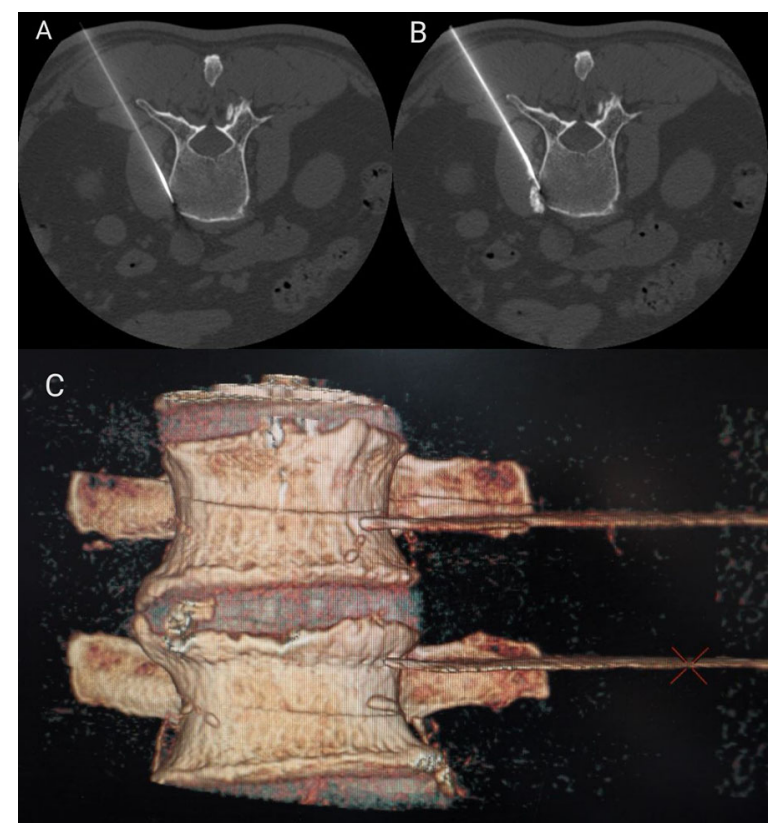

Fig. 2 Computed tomography (CT) imaging showing the position of the needle. a The needle tip is located at the anterolateral edge of the lumbar spine, $\mathbf{b}$ the diffusion position of the contrast agent covering the sympathetic ganglia, c CT three-dimensional reconstruction: the two needle tips are located at the anterolateral edge of the L2 and L3 vertebral bodies, respectively
Time-intensity curves before and 1 month after chemical lumbar sympathectomy; peak time is 72.06 and $53.53 \mathrm{~s}$, respectively

before the operation and at 2 weeks and 1, 3, 6, and 12 months after the operation. The pain score of the patients was measured using an NRS $(0=$ no pain to $10=$ worst possible pain [16]), and the patients were informed in detail before the study to ensure a more objective pain assessment. The skin temperature was measured using an infrared thermometer with an accuracy of $0.1{ }^{\circ} \mathrm{C}$ for three consecutive measurements of the soles and dorsum of the feet, and the average value was taken. A 6-min walk test was performed by those patients who were able to walk, and the number of walking patients and the distance of the 6-min walk were recorded. The healing of foot ulcers and gangrene was evaluated by a well-trained professional physician based on clinical examinations.

Pain at 1 year after CLS was analyzed according to the World Health Organization criteria for pain relief evaluation [19]. The results were divided into four grades: complete response (CR), partial response (PR), mild response, and no response. The patients with CR and PR were classified as a satisfactory efficacy group, whereas the other patients were classified as an unsatisfactory efficacy group. The adverse reactions of patients during the 
postoperative follow-up period were observed and recorded. All data collection was done by experimental researcher who were not involved in the surgery.

A sample size of 34 was needed to achieve $80 \%$ power to detect a difference of -0.3 between the null hypothesis correlation of 0.4 and the alternative hypothesis correlation of 0.7 for the correlation of preoperative TTP and NRS 1 year after CLS, using a one-sided hypothesis test with a significance level of 0.05. The sample size was calculated using the PASS version 11 statistical analysis software package.

\section{Statistical Analysis}

All continuous data with a normal distribution were presented as the mean \pm standard deviation; non-normal continuous data were described in terms of the median and quartile, and categorical variables were expressed as numbers and percentages. We used the Shapiro-Wilk normality test to graphically test the normality of the distribution of continuous variables. The correlation and partial correlation between preoperative TTP and postoperative foot pain score and skin temperature of the affected limb were analyzed. The patients were divided into two groups according to whether the efficacy of treatment was satisfactory or not 1 year after CLS. The independent sample $t$ test or Mann-Whitney $U$ test were conducted between the two groups. For categorical parameters, chisquare analysis and the Fisher's exact test were used, when appropriate. A logistic regression analysis was performed to determine the risk factors for unsatisfactory postoperative outcomes. Receiver operating characteristic (ROC) curve analysis was used to determine the diagnostic effect of preoperative TTP on the efficacy of CLS. When the $P$ value $<0.05$, the data were considered to be statistically significant. All analyses were performed using SPSS statistical software (version 23.0; IBM Corp., Armonk, NY, USA).

\section{RESULTS}

From January 2019 to July 2020, 81 patients met the inclusion criteria and successfully underwent the CEUS and CLS procedures. Of these, five patients refused the TTP examination 1 month after surgery, eight patients had subsequent lower limb vascular reconstruction surgery due to worsening condition during follow-up, five patients had limb amputation during follow-up, three patients did not provide data because of loss of contact during follow-up, and two patients died of heart disease. Overall, 58 patients were included in the final study analysis. The flow chart of the patient selection process is shown in Fig. 3.

Most variables followed a normal or approximately normal distribution. The mean age of the study population was 66.24 (range 58-78) years, and the mean BMI was 22.12 (range $18.31-26.67) \mathrm{kg} / \mathrm{m}^{2}$. Of these 58 patients, 42 (72.41\%) were men, and 27 (46.55\%) underwent CLS on the left side. The mean preoperative pain duration was 11.98 (range 7-18 ) months. Twenty-three patients (39.66\%) had a history of smoking; 20 patients $(34.48 \%)$ had ulcers and gangrene; 38 patients (65.52\%) were assessed with Fontaine class III peripheral vascular disease; and 20 patients (34.48\%) had a history of preoperative revascularization. Comorbid conditions included hypertension $(n=36, \quad 62.07 \%), \quad$ hyperlipidemia $\quad(n=40$, $68.97 \%)$, diabetes $(n=21,36.21 \%)$, coronary heart disease $(n=11,18.97 \%), \quad$ chronic obstructive pulmonary disease $(n=2,3.44 \%)$, chronic nephritis $(n=13,22.41 \%), \quad$ and rheumatoid arthritis $(n=5,8.62 \%)$. The preoperative $\mathrm{ABI}$ was $0.35 \pm 0.04$, and the preoperative tramadol dosage was $171.55 \pm 45.03 \mathrm{mg}$.

The preoperative TTP was $77.84 \pm 11.06$ (range 56-104) s, and it was significantly shortened 1 month after operation to $49.21 \pm 16.08$ (range $25-83$ ) s. The difference was statistically significant at $P<0.01$. 
81 patients of ASO-LE with moderate to severe pain

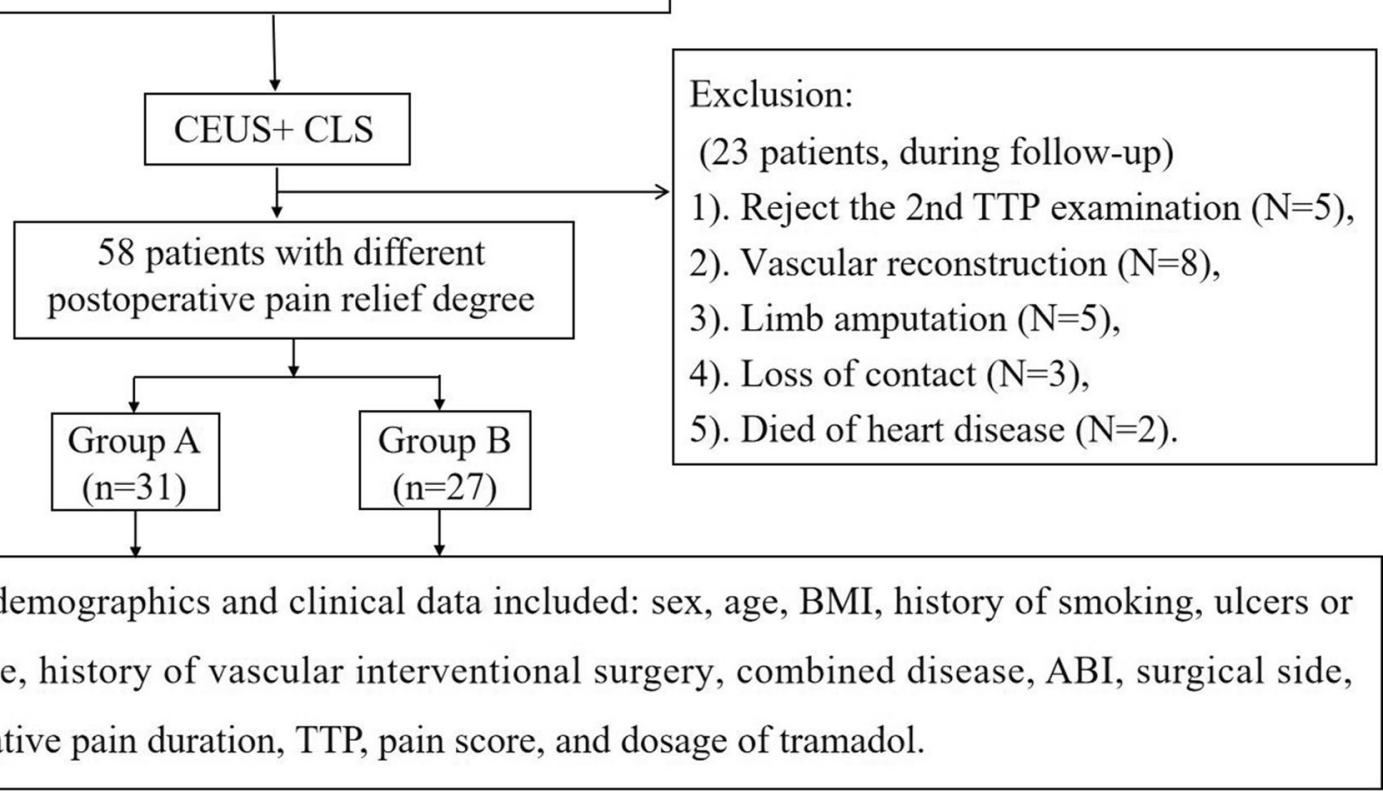

Fig. 3 Flow chart of patient enrollment. Group A (satisfactory efficacy group) consisted of patients who reported satisfaction with CLS procedure in terms of pain relief; group B (unsatisfactory efficacy group) consisted of patients who expression dissatisfaction with CLS in terms

Pain score of the lower extremity

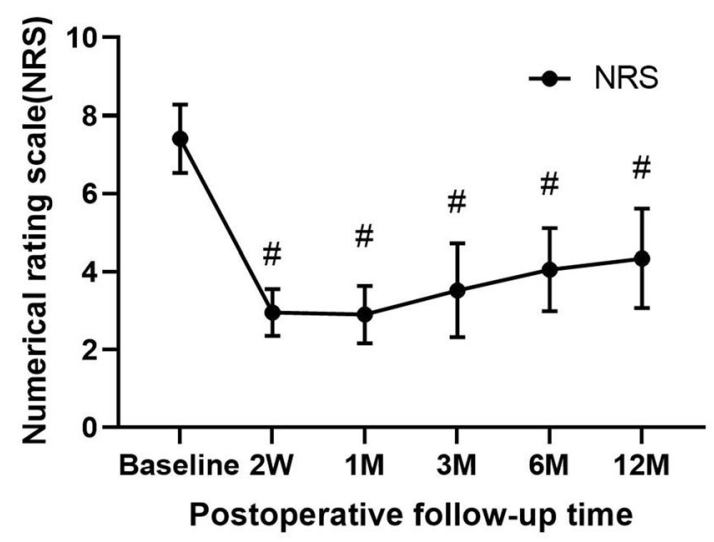

Fig. 4 Pain score and skin temperature of the affected side of the lower extremity at baseline (before surgery) and at different postoperative time points (2 weeks $[W]$ and 1,3 ,

The changes in pain score and skin temperature of lower extremity are shown in Fig. 4. The NRS measurement of the foot pain before of pain relief. $A B I$ Ankle-brachial index, ASO-LE atherosclerotic occlusive disease of the lower extremities, $B M I$ body mass index, CEUS contrast-enhanced ultrasound, CLS chemical lumbar sympathectomy, NRS numerical rating scale, TTP time to peak

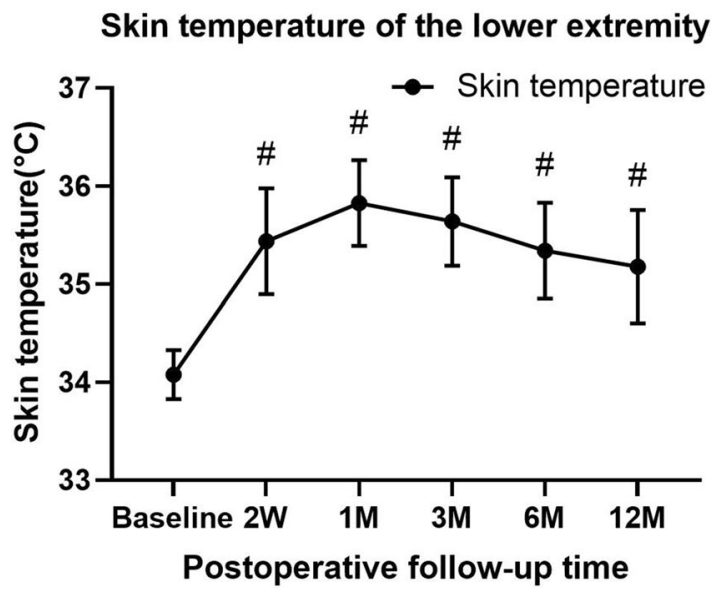

6, 12 months $[M])$. Data are presented as the mean \pm standard deviation (SD). Hashtag (\#) indicates significant difference at $P<0.01$ compared with baseline

CLS was $7.41 \pm 0.88$ and significantly decreased after CLS $(P<0.01)$ at all time points. The numbers of $\mathrm{CR}, \mathrm{PR}$, mild responses, and non- 
responses at 12 months after CLS were two (3.45\%), 29 (50.00\%), 22 (37.93\%), and five $(8.62 \%)$, respectively. The satisfaction rate of lower limb pain relief 1 year after operation was $53.45 \%(3.45+50.00 \%)$. The skin temperature of the foot before CLS was $34.07 \pm 0.25^{\circ} \mathrm{C}$, and the skin temperature significantly increased after CLS $(P<0.01)$ at all time points.

Before surgery, none of the patients were able to walk normally because of significant pain in the lower limbs; 2 weeks after CLS, eight patients $(13.79 \%)$ were able to walk a mean distance of $97.50 \mathrm{~m}$ within $6 \mathrm{~min}$. At the 1-month follow-up, the number of patients who were able to walk and the walking distance both significantly improved, with 22 patients (37.93\%) able to walk a mean distance of $160.00 \mathrm{~m}$ in $6 \mathrm{~min}$. At the 3-month and 6-month follow-ups, the number of patients who were able to walk increased to $32(55.17 \%)$, and their mean walking distance reached 199.69 and $235.63 \mathrm{~m}$ at 3 and 6 months, respectively. At the 12-month follow-up, the number of people who walked and the mean distance walked both decreased slightly; 28 patients $(48.28 \%)$ walked a mean distance of $220.36 \mathrm{~m}$. Overall, compared with preoperative data, the number of patients who were able to walk during the 12-month follow-up period after CLS and the walking distance at that time

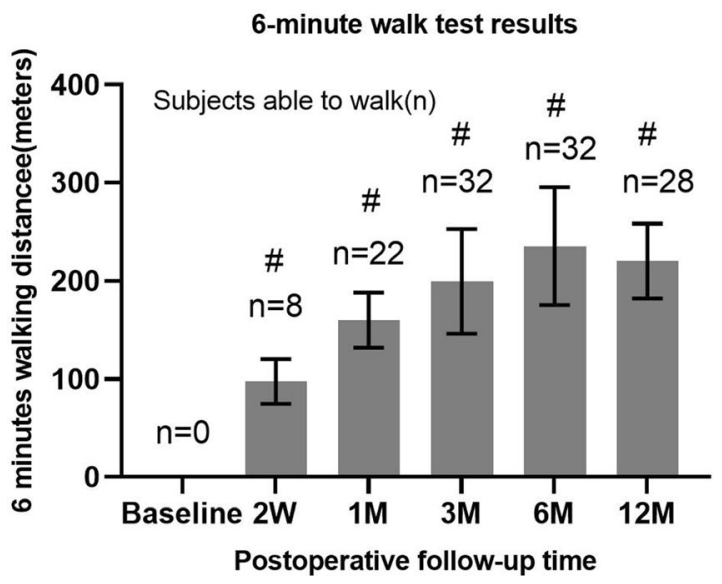

Fig. 5 Left: Results of the 6-min walk test during the follow-up period at the preoperative (baseline) time point up to the 12-month follow-up after CLS. Right: healing of ulcer or gangrene at the same time points. Time points are as in Fig. 4. Data are presented as mean \pm SD. Hashtag point had improved, with the differences being statistically significant $(P<0.01)$ (Fig. 5).

Before surgery, $20(34.48 \%)$ of the 58 patients had ischemic foot ulcers and/or gangrene. These patients also received regular dressing changes for chronic ulcer wounds. The number of patients with ulcers and/or gangrene gradually decreased throughout the follow-up period, and most ischemic ulcers ultimately had granulation tissue growth. From 6 to 12 months after CLS, the ulcers of most patients had clinically healed, although five patients had stubborn ulcers and gangrene that did not heal completely (Fig. 5).

The preoperative TTP, postoperative pain score, and skin temperature were analyzed by correlation and partial correlation analyses. Age, sex, treatment side, BMI, preoperative pain duration, and preoperative tramadol dosage were used as partial correlation control variables. Correlation analysis and partial correlation analysis indicated that preoperative TTP was positively correlated with NRS measurement at 2 weeks and $1,3,6$, and 12 months after CLS. Correlation analysis suggested that preoperative TTP was negatively correlated with skin temperature at 2 weeks and 1, 3, 6, and 12 months after CLS, whereas partial correlation analysis suggested that preoperative TTP was

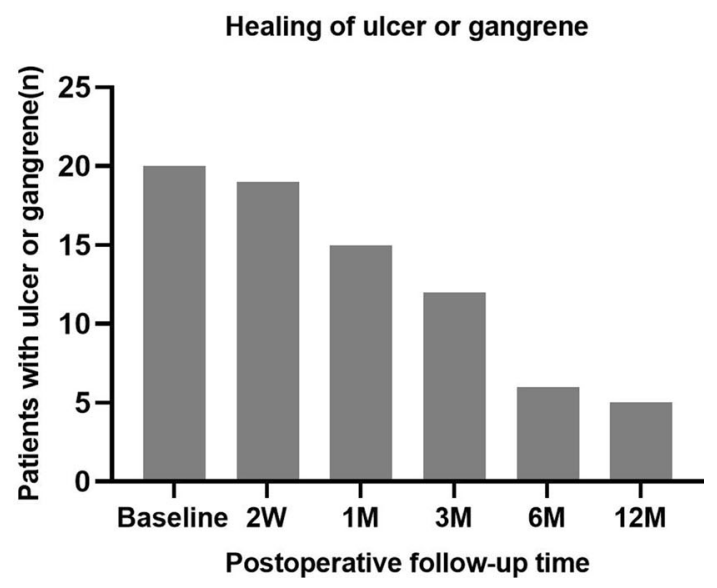

(\#) indicates significant difference at $P<0.01$ compared with baseline 
Table 1 Correlation and partial correlation analysis of preoperative time to peak intensity with postoperative numerical rating scale and skin temperatures in the lower extremities

\begin{tabular}{lllllll}
\hline Preoperative TTP & Correlation coefficient $^{\text {a }}$ & \multicolumn{6}{l}{ Postoperative time points } \\
\cline { 3 - 7 } & & 2 Weeks & 1 Month & 3 Months & 6 Months & 12 Months \\
\hline NRS & $R$ & $0.386^{*}$ & $0.422^{\#}$ & $0.354^{\#}$ & $0.413^{\#}$ & $0.475^{\#}$ \\
& $R^{\text {a }}$ & $0.438^{\#}$ & $0.506^{\#}$ & $0.509^{\#}$ & $0.517^{\#}$ & $0.551^{\#}$ \\
\multirow{2}{*}{ Skin temperatures } & $R$ & -0.230 & -0.185 & -0.218 & $-0.387^{\#}$ & $-0.457^{\#}$ \\
& $R$ & -0.248 & -0.190 & -0.241 & $-0.403^{\#}$ & $-0.437^{\#}$ \\
\hline
\end{tabular}

Control variables: Age, sex, treatment side of lower extremities, body mass index, preoperative pain duration, and preoperative dose of tramadol

NRS Numerical rating scale, TTP the time to peak intensity

${ }^{*} P<0.05,{ }^{\#} P<0.01$

${ }^{\mathrm{a}} R$, Pearson correlation coefficient; $R^{\mathrm{a}}$, partial correlation coefficient

Table 2 Comparison of demographics and other data between the two study groups according to efficacy of the chemical lumbar sympathectomy

\begin{tabular}{llll}
\hline Parameters & Group A $(\boldsymbol{n}=\mathbf{3 1})^{\mathbf{a}}$ & Group B $(\boldsymbol{n}=\mathbf{2 7})^{\mathbf{a}}$ & $\boldsymbol{P}$ value \\
\hline Sex (female/male), $n$ & $8 / 23$ & $7 / 20$ & 0.992 \\
Age $($ years) & $65.97 \pm 4.03$ & $66.56 \pm 4.10$ & 0.585 \\
BMI $\left(\mathrm{kg} / \mathrm{m}^{2}\right)$ & $22.29 \pm 2.10$ & $21.93 \pm 1.51$ & 0.459 \\
Lower extremity (left/right), $n$ & $16 / 15$ & $11 / 16$ & 0.226 \\
History of smoking $(n, \%)$ & $10(32.3)$ & $13(48.1)$ & 0.221 \\
Ulcers or gangrene $(n, \%)$ & $11(35.5)$ & $9(33.3)$ & 0.864 \\
History of vascular interventional surgery $(n, \%)$ & $8(25.8)$ & $12(44.4)$ & 0.136 \\
Combined disease $(n, \%)$ & & & \\
Hypertension & $18(58.1)$ & $18(66.7)$ & 0.501 \\
Hyperlipidemia & $19(61.3)$ & $21(77.8)$ & 0.305 \\
Diabetes mellitus & $8(25.8)$ & $13(48.1)$ & 0.077 \\
Coronary artery disease & $3(9.7)$ & $8(29.6)$ & 0.053 \\
COPD & $2(6.5)$ & $0(0)$ & 0.179 \\
Chronic nephrosis & $9(29.0)$ & $4(14.8)$ & 0.195 \\
Rheumatoid arthritis & $4(12.9)$ & $1(3.7)$ & 0.213 \\
Fontaine classification (III/IV, before CLS), $n$ & $24 / 7$ & $14 / 13$ & $0.043^{*}$ \\
Fontaine classification (II/III/IV, 1 year after CLS), $n$ & $17 / 14 / 0$ & $0 / 22 / 5$ & $<0.001^{\#}$ \\
ABI (before CLS) & $0.35 \pm 0.04$ & $0.34 \pm 0.04$ & 0.176 \\
\hline
\end{tabular}


Table 2 continued

\begin{tabular}{|c|c|c|c|}
\hline Parameters & Group A $(n=31)^{a}$ & Group B $(n=27)^{\mathrm{a}}$ & $P$ value \\
\hline $\mathrm{ABI}$ (1 year after CLS) & $0.66 \pm 0.26$ & $0.32 \pm 0.06$ & $<0.001^{\#}$ \\
\hline NRS (before CLS) & $7.26 \pm 1.00$ & $7.59 \pm 0.69$ & 0.150 \\
\hline NRS (1 year after CLS) & $3.39 \pm 0.67$ & $5.44 \pm 0.85$ & $<0.001^{\#}$ \\
\hline Skin temperature $\left({ }^{\circ} \mathrm{C}\right.$, before CLS) & $34.14 \pm 0.26$ & $34.01 \pm 0.21$ & 0.984 \\
\hline Skin temperature $\left({ }^{\circ} \mathrm{C}, 1\right.$ year after $\left.\mathrm{CLS}\right)$ & $35.54 \pm 0.41$ & $34.76 \pm 0.44$ & $<0.001^{\#}$ \\
\hline Preoperative pain duration $(\mathrm{M})$ & $11.77 \pm 2.20$ & $12.22 \pm 2.03$ & 0.426 \\
\hline TTP (s, before CLS) & $72.66 \pm 8.75$ & $83.79 \pm 10.54$ & $<0.001^{\#}$ \\
\hline TTP (s,1 month after CLS) & $36.07 \pm 7.45$ & $64.29 \pm 7.75$ & $<0.001^{\#}$ \\
\hline Tramadol dosage (mg/day, before CLS) & $177.42 \pm 42.50$ & $164.81 \pm 47.67$ & 0.292 \\
\hline Tramadol dosage(mg/day, 1 year after CLS) & $93.55 \pm 35.92$ & $153 \pm 45.84$ & $<0.001^{\#}$ \\
\hline
\end{tabular}

negatively correlated with skin temperature at 6 and 12 months after CLS (Table 1).

Patients were divided into two groups according to the efficacy of the CLS: a satisfactory efficacy group (group A) and an unsatisfactory efficacy group (group B). A comparison of demographic data and other data between the two groups is shown in Table 2. No significant differences were noted in sex, age, BMI, comorbid disease, ulcers or gangrene, history of vascular interventional surgery, preoperative $\mathrm{ABI}$, or preoperative pain duration between the groups.

No significant difference was seen in preoperative NRS results between the two groups $(P>0.05)$, but there was a significant difference in postoperative time $(P<0.05)$. NRS measurements in group B began to increase significantly 3 months after CLS, but patients in group A maintained a longer period of pain relief. A comparison of the NRS measures for the two groups is shown in Fig. 6 .

The preoperative skin temperature of the affected foot did not differ significantly between the two patient groups, but the skin temperature of patients in group A was higher than that of patients in group B at different time times after CLS $(P<0.05)$. The highest skin temperature of both groups was reached at 1 month after CLS, following which time it began to decrease. The decline in skin temperature of the affected foot of group B patients was greater than that in group A patients after 3 months $(P<0.05)$. A comparison of the skin temperature of the affected foot between two groups is shown in Fig. 6.

Patients in group A were able to walk in greater numbers and for greater distances during the 6-min walk test compared with patients in group B. At 3 and 6 months after surgery, 23 patients in group A and nine patients in group $B$ were able to walk. At 12 months after surgery, 22 patients in group A and six patients in group $\mathrm{B}$ were able to walk. Postoperative walking distance increased gradually in both groups, with the highest distance reached at 6 months after surgery $(242.61 \pm 61.51 \mathrm{~m}$ [group A] and $217.78 \pm 55.18 \mathrm{~m}$ [group B]) and decreasing 


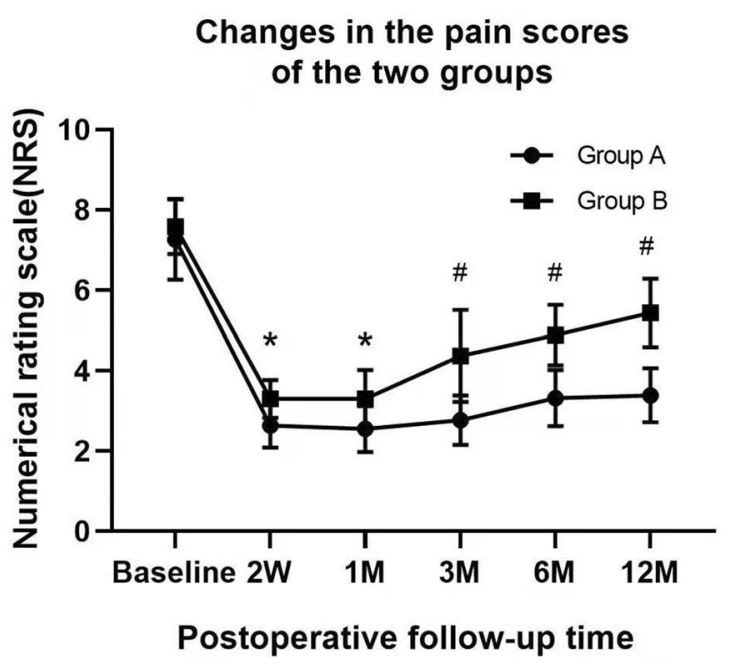

Fig. 6 Left panel: Changes in NRS before and after CLS in group A (satisfactory efficacy group) and group B (unsatisfactory efficacy group). Right panel: Changes in skin temperature of the lower limbs before and after surgery in groups A and B. Data are presented as the mean \pm SD. Time points are as in Fig. 4. Left panel: Asterisk $\left(^{*}\right)$ and hashtag $(\#)$ indicate significant difference

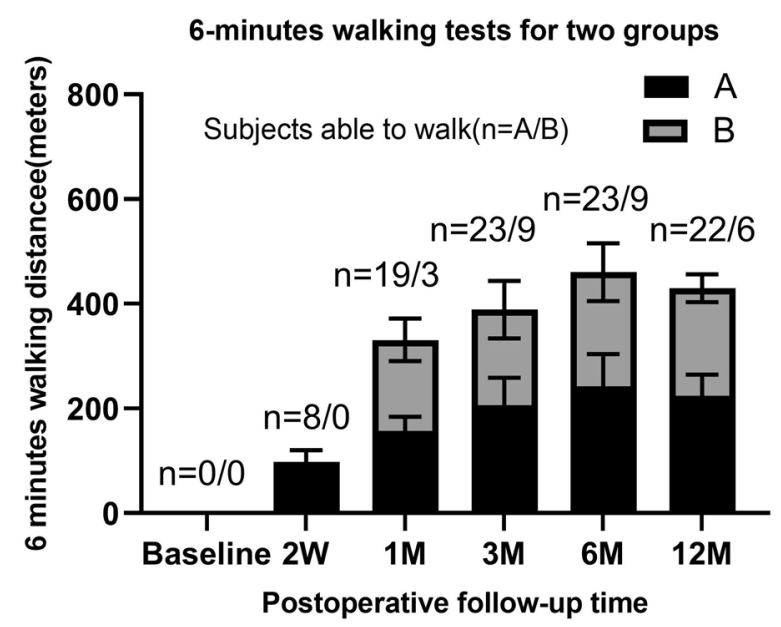

Fig. 7 Results of the 6-min walk test after CLS in group A and group B. Data are presented as the mean \pm SD. Asterisk $\left(^{*}\right)$ and hashtag $(\#)$ indicate significant difference at ${ }^{*} P<0.05$ and ${ }^{\#} P<0.01$, compared with group $B$. Time points are as in Fig. 4

slightly at 12 months $(224.55 \pm 40.21 \mathrm{~m}$ [group A] and $205.00 \pm 26.65 \mathrm{~m}$ [group B]) (Fig. 7).

Compared with preoperative data, the TTP of the two groups of patients at 1 month post
Changes in the skin temperature of the two groups

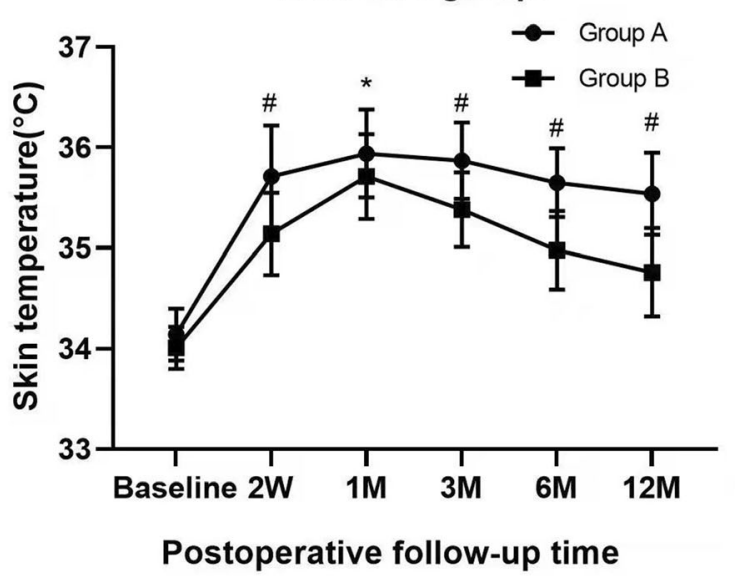

at ${ }^{*} P<0.05$ and ${ }^{*} P<0.01$, compared with group A. Right panel: Asterisk $\left(^{*}\right)$ and hashtag (\#) indicate significant difference at ${ }^{*} P<0.05$ and ${ }^{\#} P<0.01$, compared with group $B$

operation was significantly shorter $(P<0.01)$. Compared with group B, group A had a shorter TTP before the operation and at 1 month after the operation $(P<0.01)$. No significant difference was noted in the amount of tramadol used before the operation between the two groups. The amount of tramadol 1 year after the operation was significantly lower than the amount used before the operation $(P<0.001)$. Compared with group $B$, the amount of tramadol used by group A was significantly reduced 1 year after the operation $(P<0.001)$.

There was a statistical difference in the Fontaine classification between the two groups before surgery and 1 year after surgery $(P<0.05)$. Compared with patients in group $\mathrm{A}$, more patients in group $B$ had a preoperative Fontaine classification IV. One year after CLS, the number of patients with Fontaine classification IV in group A decreased to 0 , and 17 patients had transitioned to classification II; in contrast, five patients in group B remained at classification IV $(P<0.001)$ (Table 2$)$.

Binary logistic regression analysis indicated that a higher preoperative TTP was associated 


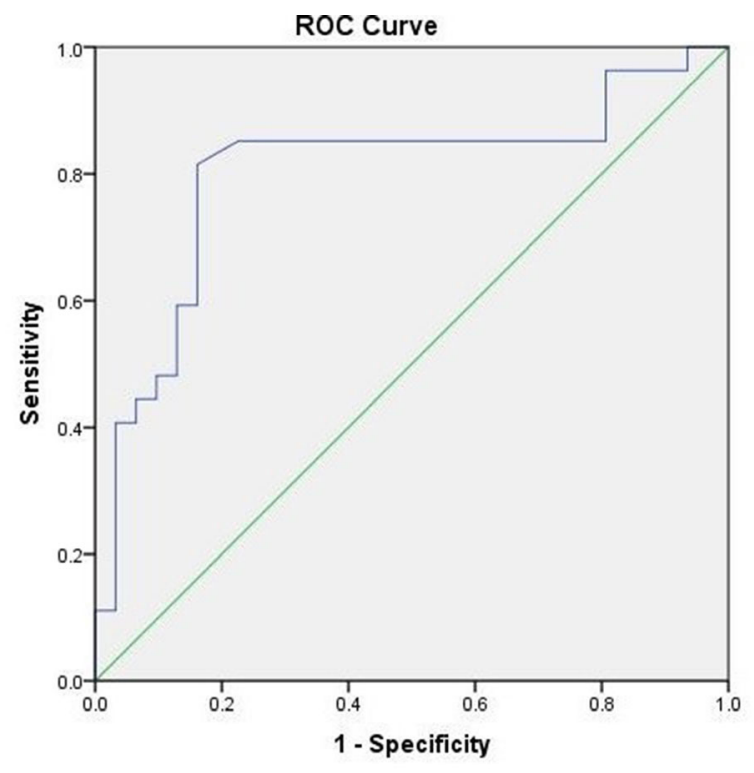

Fig. 8 Receiver operating characteristic curve of preoperative TTP to predict the effect of CLS treatment

with a higher risk of poor pain relief after CLS $(P<0.001$, odds ratio [OR] $1.126,95 \%$ confidence interval [CI] 1.058-1.205).

To predict the influence of preoperative TTP on the efficacy of CLS, an ROC curve was drawn for the preoperative TTP. The area under the curve (AUC) was 0.803 (95\% CI 0.678-0.927); the sensitivity and specificity were $81.5 \%$ and $83.9 \%$, respectively. When the preoperative TTP was $>77.5$, the risk of poor response after CLS increased (Fig. 8).

Patients were observed for 1 year after CLS to identify complications. Two patients reported testicular pain on the affected side immediately after the injection of ethanol, but the pain disappeared on day 2 after administration of tramadol (Grunenthal GmbH, Stolberg, Germany). Four patients reported anterolateral thigh pain and numbness that resolved 1 to 2 weeks after treatment with gabapentin (Jiangsu Hengrui Pharmaceutical Co., Ltd., Lianyungang, Jiangsu, China) and mecobalamin (Eisai [Liaoning] Pharmaceutical Co., Ltd. [China], Benxi City, Liao Ning Province, China). No patients had urination, ejaculation, or bowel dysfunction.

\section{DISCUSSION}

Previous studies $[7,8]$ have shown that CLS can effectively treat lower limb ischemic diseases. Alexander et al. [7] conducted a follow-up observation of 544 patients with severe ischemic lower extremity disease after CLS for up to 13 years. These authors reported that $72 \%$ of lower limb symptoms improved significantly and that the postoperative improvement was mainly based on three evaluation indicators: the relief of resting pain, improvement of skin blood flow, and healing of ischemic ulcers.

However, for patients with severe ischemic lower limbs with ulcers or gangrene, the effect of CLS sympathetic denervation on lower limb vasodilation is uncertain $[8,20]$. Tay et al. [20] studied 119 patients with peripheral vascular disease who were treated with CT-guided CLS and found that $30.3 \%$ showed improvement within 3 months after surgery, with improvement defined as doubling of the walking distance, no resting pain, or ulcer healing; however, the improvement rate among patients with ulcers or gangrene was only $19 \%$. Dreyfus et al. [8] studied 162 patients with ischemic pain in the lower limbs who were treated with CLS and found that 92 patients had sustained improvement at 1 month and 1 year after surgery; however, for patients with combined intermittent claudication and leg skin ulcers, the effectiveness of the surgery was relatively poor.

These results have also been confirmed in our clinical practice, and are rather confusing. Patients with ulcers and gangrene are mainly patients with end-stage ASO-LE; therefore, it is of great significance to determine the predictors of CLS treatment effect in these patients.

To determine these predictive factors, we must understand the mechanism of CLS. The vasodilation effect of CLS is most directed towards promoting the establishment of small blood vessels and collateral circulation. Experts with many years of treatment experience generally believe that the effect of CLS treatment is directly related to the level of skeletal muscle perfusion and the number of collateral vessels. This is also consistent with the conclusions of 
Holiday et al. [21], who stated that CLS is more successful in patients with arterial ischemia that is not extensive, in whom collateral circulation is basically established, blood flow meters can detect pulsating blood flow, and clinical manifestations tend to improve. For patients with a longer course of disease and more severe disease, because the ischemic area after CLS lacks collateral vessels for expansion, more blood perfusion is obtained and the arteries at the proximal end of the occluded blood vessels will expand. More blood will aggravate the degree of ischemia in the occluded vascular area, which will result in an unsatisfactory curative effect or will aggravate the condition. It can be inferred that a better CLS treatment effect needs to be established with a certain reserve of peripheral perfusion and collateral vessels.

ASO-LE consists of two parts: arterial stenosis of the lower extremities and chronic ischemia of the skeletal muscles. Bragadeesh et al. [22] showed that CEUS can assess the severity of ASO-LE by measuring skeletal muscle blood flow reserve. These authors used canine models of lower limb arterial stenosis to observe the skeletal muscle perfusion of the quadriceps muscle at rest and after contraction during exercise or from human adenosine injection. After exercise or adenosine injection, the blood flow reserve of skeletal muscle decreased significantly with increasing severity of arterial stenosis-and these changes can be reflected in CEUS. Unlike other currently available diagnostic methods, CEUS can reflect the perfusion and collateral circulation of the skeletal muscles of the limbs and can more accurately reflect the state of the microvessels.

In addition, Song et al. [12] observed the perfusion of the skeletal muscle of the lower extremity ASO-LE by CEUS, and assessed the collateral circulation by CT angiography. Through correlation analysis, this research team found that TTP in CEUS could be used to evaluate the perfusion disorder of calf skeletal muscle and that it had a good correlation with the degree of collateral circulation.

Our results show that preoperative TTP was significantly correlated with the postoperative pain score and skin temperature. In addition to pain relief and increased skin temperature, the numbers of people able to walk, the walking distance, and ulcer and gangrene healing significantly improved after CLS. Moreover, the Fontaine classification improved and was statistically different between the two subgroups $(P<0.05)$. Preoperative TTP thus appears to correlate well with the number of people able to walk, the walking distance, ulcer healing, and changes in Fontaine classification.

The preoperative TTP in the group with satisfactory pain relief was shorter, indicating that this group of patients had better calf skeletal muscle perfusion and collateral circulation. This result confirms that CLS efficacy relies, to a certain degree, on skeletal muscle perfusion and collateral circulation. The TTP is the main research index of this study, and it may be affected by individual circulatory function, heart function, hypertension, hyperlipidemia, smoking, age, and other factors. However, no statistically significant differences were found in these risk factors between the two groups.

This study has some limitations. First, although some research has shown that CLS can achieve the same therapeutic effect as open lumbar sympathectomy [23], CLS is based on CT images; as such, there is no visual field, and the number, location, and shape of the sympathetic ganglia may vary. Thus, CLS may not completely destroy the sympathetic ganglia. Second, the NRS is a relatively subjective assessment of pain. Although educational guidance was provided before the study, differences in age and cultural background of the patients may have affected the effect of this guidance. Also, the temperature measurement may have been affected by the external environment, resulting in errors, and the area of the ulcers was not used as an observation index for quantitative evaluation. In addition, this study only included 58 patients, a relatively small population size. Some excluded patients underwent vascular reconstruction surgery or lower limb amputation as a result of physical causes, and most of these patients had severe Fontaine IV classification. A multi-center study with a large sample is needed to verify the results of this study. Because this study only involved patients with ASO-LE, other lower 
extremity ischemic diseases must be explored in future studies.

\section{CONCLUSIONS}

The TTP obtained by CEUS was able to predict the therapeutic effect of CLS on patients with end-stage ASO-LE. When the preoperative TTP was $>77.5$, the risk of poor response after CLS increased. Although we recognize the limitations of this study, we believe that the positive results described here are worth continued study and will provide new guidance on the predictive effect of CEUS for CLS use in endstage ASO-LE.

\section{ACKNOWLEDGEMENTS}

We thank all patients who participated in the study and all of the research staff (Yue $\mathrm{Wu}$, Suming Tian,Chunye Li, Weibo Zhang, Qianqian Xing, Gang Chen). We thank Shiyan Li, Xueyao Yin, and Zhengfeng Zhou for their guidance on experimental methods and statistical methods.

Funding. This work and the Rapid Service Fee were funded by Health and Family Planning Commision of Zhejiang Province (No. 2019332871).

Authorship. All named authors meet the International Committee of Medical Journal Editors (ICMJE) criteria for authorship for this article, take responsibility for the integrity of the work as a whole, and have given their approval for this version to be published.

Authorship Contributions. All authors contributed to data analysis, drafting or revising the article, gave final approval of the version to be published, and agree to be accountable for all aspects of the work.

Disclosures. Yue $\mathrm{Wu}$, Suming Tian,Chunye Li, Weibo Zhang, Qianqian Xing, and Gang Chen declare that they have nothing to disclose.
Compliance with Ethics Guidelines. This study was performed in accordance with the Helsinki Declaration of 1964, as revised in 2013, and its protocol was approved by the Institutional Review Committee of Run Run Shaw Hospital of Zhejiang University in Hangzhou, Zhejiang Province, China. Data collection was pre-planned and registered with the Chinese Clinical Trial Registry (Registration number: ChicTR1900028424). All participants were informed about the risks and complications before surgery, and all patients provided written informed consent.

Data Availability. The datasets generated during and/or analyzed during the current study are available from the corresponding author on reasonable request.

Open Access. This article is licensed under a Creative Commons Attribution-NonCommercial 4.0 International License, which permits any non-commercial use, sharing, adaptation, distribution and reproduction in any medium or format, as long as you give appropriate credit to the original author(s) and the source, provide a link to the Creative Commons licence, and indicate if changes were made. The images or other third party material in this article are included in the article's Creative Commons licence, unless indicated otherwise in a credit line to the material. If material is not included in the article's Creative Commons licence and your intended use is not permitted by statutory regulation or exceeds the permitted use, you will need to obtain permission directly from the copyright holder. To view a copy of this licence, visit http://creativecommons.org/licenses/by$\mathrm{nc} / 4.0 /$.

\section{REFERENCES}

1. Dua A, Lee CJ. Epidemiology of peripheral arterial disease and critical limb ischemia. Tech Vasc Interv Radiol. 2016;19:91-5.

2. Jude EB, Eleftheriadou I, Tentolouris N. Peripheral arterial disease in diabetes-a review. Diabet Med. 2010;27:4-14. 
3. Takahara M, Kaneto H, Katakami N, Iida O, Matsuoka T, Shimomura L. Effect of sarpogrelate treatment on the prognosis after endovascular therapy for critical limb ischemia. Heart Vessels. 2014;29: $563-7$.

4. Chalmers N, Walker PT, Belli AM, et al. Randomized trial of the SMART stent versus balloon angioplasty in long superficial femoral artery lesions: the SUPER study. Cardiovasc Interv Radiol. 2013;36:353-61.

5. Bhattarai BK, Rahman TR, Biswas BK, Sah BP, Agarwal B. Fluoroscopy guided chemical lumbar sympathectomy for lower limb ischemic ulcers. J Nepal Med Assoc. 2006;45:295-9.

6. Nesargikar PN, Ajit MK, Eyers PS, Nichols BJ, Chester JF. Lumbar chemical sympathectomy in peripheral vascular disease: Does it still have a role? Intern J Surg. 2009;7:145-9.

7. Alexander JP. Chemical lumbar sympathectomy in patients with severe lower limb ischaemia. Ulster Med J. 1994;63:137-43.

8. Dreyfus A, Fondras JC, Loubrieu G, Ntarundenga U. Our experience of lumbar chemical sympathectomy in arterial occlusive diseases of the lower limbs. Agressologie. 1990;31:207-9.

9. Lindner JR, Womack L, Barrett EJ, et al. Limb stressrest perfusion imaging with contrast ultrasound for the assessment of peripheral arterial disease severity. JACC Cardiovasc Imaging. 2008;1:343-50.

10. Duerschmied D, Zhou Q, Rink E, et al. Simplified contrast ultrasound accurately reveals muscle perfusion deficits and reflects collateralization in PAD. Atherosclerosis. 2009;202:505-12.

11. Amarteifio E, Wormsbecher S, Krix M, et al. Dynamic contrast-enhanced ultrasound and transient arterial occlusion for quantification of arterial perfusion reserve in peripheral arterial disease. Eur J Radiol. 2012;81:3332-8.

12. Song Y, Li Y, Lu W, Gao Y. Preliminary study of contrast-enhanced ultrasound in correlation between muscle perfusion deficits in lower extremity arterial disease and its collateralization. Zhonghua Yi Xue Za Zhi. 2014;94:507-9.

13. Kaspar M, Partovi S, Aschwanden M, et al. Assessment of microcirculation by contrast-enhanced ultrasound: a new approach in vascular medicine. Swiss Med Wkly. 2015;145:w14047.

14. Kalliokoski KK, Laaksonen MS, Takala TO, Knuuti J, Nuutila P. Muscle oxygen extraction and perfusion heterogeneity during continuous and intermittent static exercise. J Appl Physiol. 2003;94:953-8.

15. Ledermann HP, Schulte AC, Heidecker HG, et al. Blood oxygenation level-dependent magnetic resonance imaging of the skeletal muscle in patients with peripheral arterial occlusive disease. Circulation. 2006;113:2929-35.

16. Levy N, Mills P, Rockett M. Post-surgical pain management: time for a paradigm shift. $\mathrm{Br} \mathrm{J}$ Anaesth. 2019;123:e182-6.

17. Tao S, Yu A, Yu G, et al. Anatomic study and clinical application of sural neuron-myocutaneous compound flap transposition. Chin J Plast Surg. 2008;24:16-9.

18. Zechlinski JJ, Hieb RA. Lumbar sympathetic neurolysis: how to and when to use? Tech Vasc Interv Radiol. 2016;19:163-8.

19. Ding Y, Peng Y, Li H, Zhao R, Zhao G. Evaluation of combined radiofrequency and chemical blockade of multi-segmental lumbar sympathetic ganglia in painful diabetic peripheral neuropathy. J Pain Res. 2018;11:1375-82.

20. Tay VK, Fitridge R, Tie ML. Computed tomography fluoroscopy-guided chemical lumbar sympathectomy: simple, safe and effective. Australas Radiol. 2002;46:163-6.

21. Holiday FA, Barendregt WB, Slappendel R, Crul BJ, Buskens FG, van der Vliet JA. Lumbar sympathectomy in critical limb ischaemia: surgical, chemical or not at all? Cardiovasc Surg. 1999;7:200-2.

22. Bragadeesh T, Sari I, Pascotto M, Micari A, Kaul S, Lindner JR. Detection of peripheral vascular stenosis by assessing skeletal muscle flow reserve. J Am Coll Cardiol. 2005;45:780-5.

23. Piatko VE, Sukhotin SK. Chemical lumbar sympathectomy in patients with chronic ischemia of the lower extremities. Anesteziol Reanimatol. 2004;4: 31-3. 